\title{
Updation of Cartographical Database with the Aid of Different Traits in Blending of ANN and ANFIS
}

\author{
Priti Tyagi \\ Associate Professor,
}

\author{
Udhav Bhosle, Ph.D \\ Principal, Rajiv Gandhi Institute of Technology \\ Mumbai University
}

\begin{abstract}
In recent years, the image processing plays a major role in real world and that are utilized in many of the application domains. Besides the other, in the field of remote sensing the contribution of images is thunderstruck. Nowadays, a wide number of buildings are updated often; this can be updated in the cartographical database which is comprised of remote sensing images of buildings. Updating cartographic database is an important process in order to identify the new buildings and roads. In this work, the high resolution satellite images are utilized to identify the updated buildings. For this process, images of different time period is utilized and then the traits are identified; here the traits are building, road and vegetation. For this intention, the Artificial Neural Network (ANN) is designed separately for each of them. Once the traits are identified from both images of different time period, then the unique traits are identified. Prior to this process, with the aid of the ANFIS the each region wise traits are trained. Subsequently, the uniquely identified traits are evaluated in this ANFIS in order to identify whether the trait is building or not. Once the building is identified, then it is marked in the original image and then the image is utilized to update in the cartographical database.
\end{abstract}

\section{Keywords}

cartographical data, Artificial Neural Network (ANN), traits, building detection, ANFIS.

\section{INTRODUCTION}

Over the last decade research towards the automatic extraction of buildings and other man-made objects from aerial and satellite imagery has gained considerable attention. Processing schemes and systems of the literature, curve propagation techniques like snakes, active contours, deformable models and more recently level sets have exposed capable results among various methods [6]. A significant new data source for building extraction is provided by the high-resolution satellite imagery [8]. From high spatial-resolution remote sensing images, automatic building detection has gained extensive attention for GIS (Geographic information system) data production [10] three-dimensional urban visualization, urban intra-structure analysis [11], homeland security, disaster management and hazard damage evaluation [7]. This process is carried out particularly in many geospatial applications like urban city design and planning, military simulation, and site monitoring of a particular geographic location [19] [9].

Unfortunately, to manually label buildings in a given aerial or satellite image for numerous reasons is tedious for a human expert. Initially, the buildings may be imaged from different viewpoints. They may not possess an exceptional representation. Second, with the environment like occlusion by trees the buildings may have compound interaction. In addition, they may occlude each other [13]. Third, to detect buildings consistently, the illumination and contrast in the image may not be adequate. Fourth, with many buildings in them these images may cover large geographic areas. Analyzing the image may take time. Finally, buildings do not comprise standard size and shape [17]. And a poor detection performance is exhibited by many existing building detection techniques that depend principally on color information [2]. Thus, on VHR aerial and satellite images, development of robust and fast building detection algorithms has become a inevitable [18].

With resolution higher than 5 meters, building recognition requires high-resolution aerial photographs or satellite images, which can express buildings as objects instead of mixed pixels [1] [5]. Before performing building renovation, the majority of researches detect the locations of buildings while the ground plane is not accessible or the existing databases is the probable occurrence of errors as well as their potential lack of authenticity and incompleteness [4]. There are two main problems that needs to be solved in any buildings detection approach. From the background the interested objects need to be segmented, and the fragmented line segments of the interested objects should be grouped to human-made structures. [3]. To detect buildings according to the range measuring data many different methods are used [16]. For instance, the data fusion of CIR (colored infrared) imagery and Light Detection and Ranging (LiDAR) data for Classification technique can be used to separate buildings from other categories [12][20]. With metric resolution in the field of building detection in urban areas, the huge amount of optical and SAR data, provided by current satellite sensors, offers today new perspectives and challenges [15][14].

\section{RELATED RESEARCHES: A REVIEW}

Sirmacek et al. [1] have proposed a method. This method is proposed based on local feature point extraction using Gabor filters. There may be some errors in the operation. Second, the urban area is dynamic. So, detection should be done occasionally since this is time consuming. An automated system is required to detect the urban area from aerial and satellite images to handle these shortcomings. To vote for the candidate urban areas they have used these local feature points. Then using an optimal decision-making approach on the vote distribution they have detected the urban area. At this point they have tested this method on a varied panchromatic aerial and Ikonos satellite image set. In practical applications, the test results specify the possibility of using this method.

Sirmacek et al. [2] have proposed a building detection method. This method was developed using local feature vectors and a probabilistic framework. Four different local feature vector extraction methods was initially introduced. Extracted local feature vectors serve as interpretation of the probability density function to be anticipated. In other words, as joint random variables they have represented building 
locations in the image. Using the modes of the estimated density, they have detected building locations in the image, in addition to other probabilistic properties, but introduced data and decision fusion methods. These are estimated based on probabilistic framework to identify the building locations. They have used pick certain crops of VHR panchromatic aerial and Ikonos satellite images to this method. Here they have assumed that these crops are detected using previous urban region detection method. By two different sensors the test images are acquired, and they have different spatial resolutions. Also, buildings in these images have varied characteristics. Hence, to automatically detect buildings in a robust and fast manner in Ikonos satellite and aerial images, wide-ranging tests indicated that the method can be used.

Theng [3] have discussed the growth of an active contour model initialization algorithm. To compute the snake contours, the prototype uses the existing improved snake energy function, but instead of radial casting algorithm it initializes the model with circular casting algorithm. Without analysis of accuracy and structure details they have used large detached buildings. From digital images are building detection and building reconstruction, the most important tasks in building extraction. To extract well-structured buildings a point process technique was developed.

Sirmacek et al.[4] have proposed a building detection method. This proposed method is developed using local feature vectors and a probabilistic framework. Initially they established four different local feature vector extraction methods. Extracted local feature vectors serve as observations of the probability density function to be estimated. In other words, in the image, as joint random variables they have represented building locations. They detect building locations in the image, using the modes of the estimated density, as well as other probabilistic properties. Based on their probabilistic framework to detect building locations they have also introduced data and decision fusion methods. To test their method they have picked certain crops of VHR panchromatic aerial and Ikonos satellite images. By two different sensors they have assumed that these crops are detected using their previous urban region detection method. Their test images are acquired, and they have different spatial resolutions. For that reason, on a diverse data set they have tested their methods. Extensive tests indicated that to automatically detect buildings in Ikonos satellite and aerial images their method can be used.

Sirmacek et al.[5] have proposed the use of scale invariant feature transform (SIFT) and graph theoretical tools. In detecting objects under various imaging conditions SIFT keypoints are powerful. However, for detecting urban areas and buildings alone SIFT is not sufficient. Therefore, in terms of graph theory they formalize the problem. They have represented each keypoint as a vertex of the graph, in forming the graph. Between these vertices like spatial distance and intensity values, the unary and binary relationships lead to the edges of the graph. They extracted the urban area using multiple subgraph matching method, based on this formalism. Then, using graph cut method they have extracted separate buildings in the urban area. Using panchromatic 1-mresolution Ikonos imagery they have formed a diverse and representative test set. They have reported very promising results on automatically detecting urban areas and buildings by extensive testings.

Xian-Zhang Pan [6] has proposed the article, building coverage ratio (BCR) and floor area ratio (FAR) values. These values are extracted from high resolution satellite images and were used to point out buildings' stretching on the surface and growth along the third dimension within areas of interest in Shanghai City, P.R. China. While the traditional commercial areas have higher FAR and BCR ratios the variation of FAR is higher than that of BCR in the inner circle, and that the newer commercial centers have higher FAR and lower BCR values which is proved from the results. The land resources are inefficiently used by that both older building areas and villa areas is also suggested by the results. Therefore for those fast economic growing regions, better planning and management of urban land are needed.

Awrangjeb et al. [7] have presented an automatic system. The system is developed for the recognition of buildings from LIDAR data and multispectral imagery that employs a threshold-free assessment system that does not engage any thresholds. Based on human choice the proposed system is modeled. A 'primary building mask' and a 'secondary building mask' are the two binary masks obtained from the LIDAR data. Using the normalized difference vegetation index derived from orthorectified multispectral images, line segments are extracted from around the primary building mask, the segments around trees being removed. Initial building positions are obtained based on the remaining line segments. The proposed threshold-free evaluation system makes one-to-one correspondences using nearest centre distances between detected and reference buildings. A total of 15 indices are used to indicate object-based, pixel-based and geometric accuracy of the detected buildings. It is experimentally shown that the proposed technique can successfully detect rectilinear buildings, when assessed in terms of these indices including completeness, correctness and quality.

\section{PROPOSED WORK}

Updating the building databases with the aid of high resolution SAR images is carried out in this work. For achieving this, these images are utilized in order to improve the cartographic database and here the proposed research is comprised of 3 phases that are traits identification, region wise feature training and the detection of new building. Initially, in the traits detection phase, the traits road, building and the vegetation spot is identified with the aid of 3 different ANN. Subsequently, the image is trained by utilizing its region wise measurements in order to identify the building and in the new building detection phase the new building is detected with the aid of the aforesaid phases. The upcoming sessions details the process.

\subsection{Traits Identification}

In this phase, the road, vegetation and the building in the image are the traits which are identified with the aid of 3 separate ANNs. Initially, the traits are identified manually and then some of the appropriate features are extracted from the image and then they are trained in the ANNs. In this work, we utilize two images of the identical spot in which the current version of the spot and also the previous version which is utilized to identify the updation of building in the satellite images. Let $I_{t 1 \text { and }} I_{t 2}$ both are satellite images of different time period which is of dimension $I \times J$ in which $0 \leq i \leq I-1,0 \leq j \leq J-1$ from the database $D$. These both images are obtained in different time periods of 
the identical spot as discussed earlier. Let $p_{i j}$ be the pixels of the image and the features contrast, homogeneity, mean, variance, $R, G, B$ values of each pixel of the identified spot (either road or building or vegetation) in the image is extracted and trained in 3 different networks.

The features extracted from the image are contrast, mean, homogeneity, variance and the pixel's $\mathrm{R}, \mathrm{G}, \mathrm{B}$ values also extracted from the pixel $p_{i j}$

$$
\begin{gathered}
\mu=\sum_{i=0}^{I-1} \sum_{j=0}^{J-1} \frac{p_{i j}}{I x J} \\
c=\sum_{i=0}^{I-1} \sum_{j=0}^{J-1}|i-j| p(i, j) \\
h=\sum_{i=0}^{I-1} \sum_{j=0}^{J-1} \frac{p(i, j)}{1+|i, j|}
\end{gathered}
$$

$$
\sigma^{2}=\frac{\sum_{i=0}^{I-1 J-1} \sum_{j=0}^{J}\left|p_{i j}\right|^{2}-\frac{\left|\sum_{i=0}^{I-1} \sum_{j=0}^{J-1} p_{i j}\right|^{2}}{I x J}}{I * J-1}
$$

The features are extracted from the image as stated above and then these features are trained in different ANNs in order to identify the road, building and the vegetation. The neural network $N$ is trained with the assist of image's pixels contrast, homogeneity, mean, variance and the RGB values. Networks are generated separately for identifying the road, building and the vegetation respectively. These three networks are generated with 7 inputs (identical for the $3 \mathrm{ANNs}$ ) and the outcome is different depends upon the traits (either road or building or vegetation).

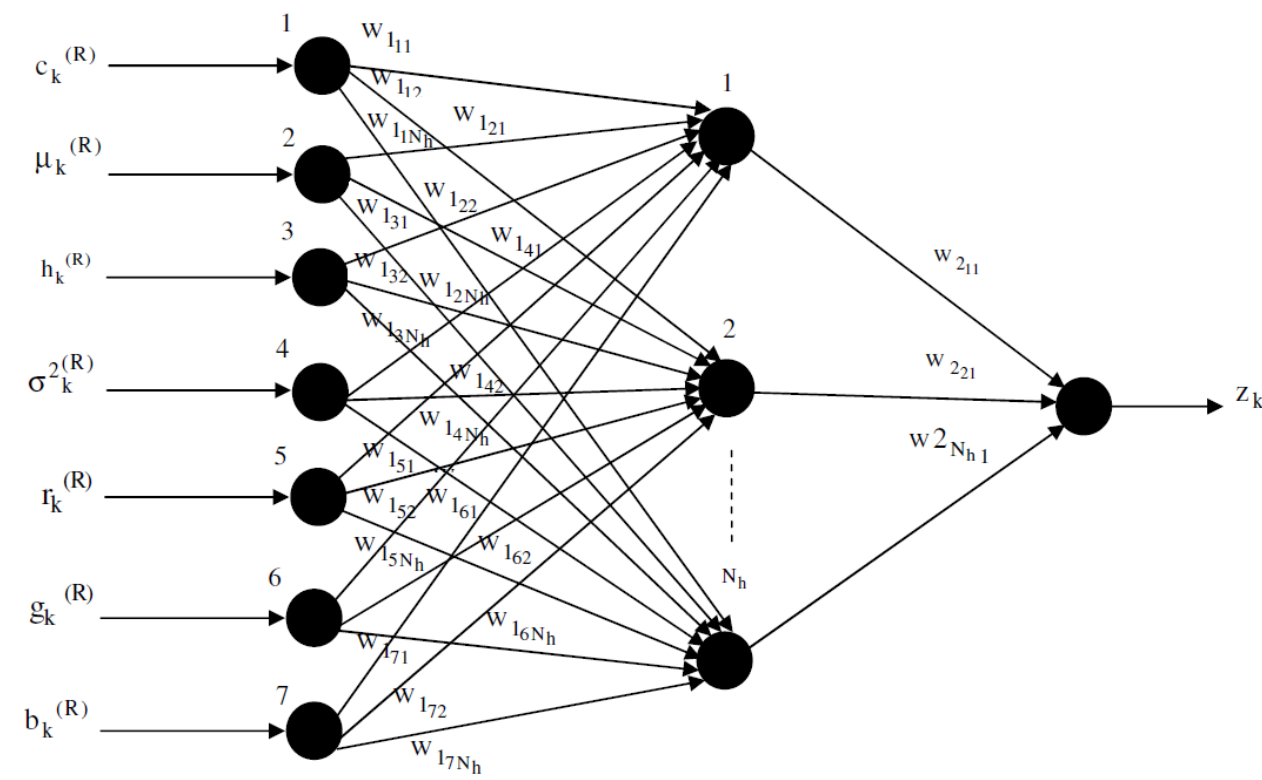

Fig 1: Broad view of the Generated ANN for identifying the traits (road, building and Vegetation)

The generated ANN is common form to identify all these traits the difference between them is, in the road trait identifier, the roads are detected manually and the corresponding regions are extracted with the aforementioned 7 features and trained in the network and the output may set to as well as for the building and vegetation traits are also identified in the separate networks. The minimization of error is reduced by the back propagation algorithm.

a) Initially, the weights are assigned to the hidden layer neurons. The input layer has a constant weight, whereas the weights for output layer neurons are chosen randomly. Then the bias function and the output layer activation function are computed by using the eqns (5) and (6)

$$
Z_{k}=\alpha+\sum_{l=0}^{N_{h}-1}\left(\begin{array}{l}
w_{l k} c_{l k}^{(R)}+w_{l k} \mu_{l k}^{(R)}+w_{l k} h_{l k}^{(R)} \\
\left.+\sigma^{2} l k w_{l k}^{(R)}+w_{l k} r_{l k}^{(R)}+w_{l k} g_{l k}^{(R)}+w_{l k} b_{l k}^{(R)}\right)
\end{array}\right)_{(5)}
$$

$$
\begin{gathered}
H\left(Z_{k}\right)=\frac{1}{1+e^{-Z_{k}}} \\
H\left(Z_{k}\right)=Z_{k}
\end{gathered}
$$

b) Then, the back propagation error is computed for each node and the weights are updated by using the eqn (8)

$$
E r r=\frac{1}{N_{h}} \sum_{o=0}^{N_{h}-1} E_{o}-Z_{o}
$$

Adjust the weights of all the neurons when the BP error is determined as follows,

$$
w_{l k}=w_{l k}+\Delta w_{l k}
$$

The change in weight $\Delta w_{l k}$ can be determined as 


$$
\Delta w_{l k}=\gamma \cdot z_{l k} \cdot E r r
$$

$E r r$ is the BP error and $\gamma$ is the learning rate and it normally ranges from 0.2 to 0.5 .

(4) After adjusting the weights, repeat steps (5) and (6) until the BP error gets minimized.

Normally, it is repeated till the criterion, Err $<0.1$ is satisfied.

Once the error gets minimized to minimum value it is construed that the designed network is well trained for its further testing phase and the BP algorithm is terminated. Thus

the 3 networks are trained for both $I_{t 1}$ and $I_{t 2}$ images in order to identify the traits road, building and the vegetation. In the testing phase if the image is given to these generated networks this may identified the building, road and vegetation traits.

Subsequent to the traits identification process, from the

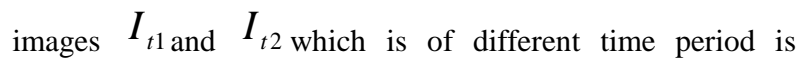
analyzed and the uncommon traits are extracted and then they are used as the traits here. After that, a mask $M$ is generated with the same dimension of image i.e $I \times J$. In the mask the identified regions are applied with the following sequence of steps in order to eliminate the unwanted spots.

1. Firstly, the identified traits are marked in the mask $M$ which are the road, building and the vegetation traits are identified which are detected in section 3.1.

2. Subsequent to the aforementioned process, the uncommon spots is identified in the mask $M$ and it is indicated by $B$ that is a vector of uncommon spots. (For example the vegetation area may now changed as buildings or roads)

3. After that, the identified uncommon spots $B$, s region property area is computed which is the number of pixels in the area is computed and a thresholding is applied in order to eliminate the small spots.

4. Consequently, the morphological operation 'imclose' is applied which closes the image morphologically with the utilization of the structural element.

5. The binary form of the original image is utilized to detect the neighbor pixels of the uncommon spots $B$ which are done by determining difference between the coordinates of the binary image and the uncommon spots $B$. This uncommon spots $B$ is utilized to determine the updation of new buildings in the current SAR image.

\subsection{Region wise Feature Training through ANFIS}

In this phase, the updation of new building is identified and masked in the original current version of image. For this process, the manually identified regions such as road, building and the vegetation are utilized. The region properties are computed in order to train it in the ANFIS networks which are convex area, eccentricity, extent and orientation. They are detailed below.

Convex area $c v$-Number of pixels in the convex image
Eccentricity $e c$ - A value that specifies the eccentricity of the ellipse that has the same second-moments as the region. The eccentricity is the ratio of the distance between the foci of the ellipse and its major axis length.

Extent $e x$ - the ratio of pixels in the region to pixels in the total bounding box. Computed as the Area divided by the area of the bounding box

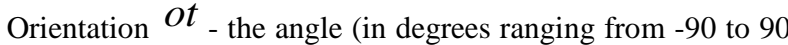
degrees) between the x-axis and the major axis of the ellipse that has the same second-moments as the region.

The aforementioned properties of the regions are utilized to train the ANFIS which is utilized to identify whether the identified uncommon spots are either building or road or vegetation. In this phase, the training of ANFIS is done with the utilization of different regions (building or road or vegetation). ANFIS is a class of adaptive networks that may acts as a fundamental framework for adaptive fuzzy inference systems [29]. For the sake of simplicity we suppose our 4 inputs are $c v, e c$, $e x$ and $o t$. The output of the ANFIS network is single which is $Y$. Each input has two fuzzy sets $\mathrm{A} 1, \mathrm{~A} 2$ and $\mathrm{B} 1, \mathrm{~B} 2$ and $\mathrm{C} 1, \mathrm{C} 2$ and D1, D2. Each circle shows a fixed node, whereas every square indicates an adaptive node. So the rule base system has sixteen if-then rules of Takagi-Sugeno's type as follows,

Rule $i$ : If cv is $A_{i}$, ec is $B_{i}$, ex is $\mathrm{C}_{\mathrm{i}}$ and ot is $\mathrm{D}_{\mathrm{i}}$

then $\mathrm{f}_{\mathrm{i}}=p_{i} c v+q_{i} e c+s_{i} e x+t_{i}$ ot $+u$

$u=1,2,3,4$

where $f_{i \text { is the output and }} p_{i}, q_{i}, s_{i}, t_{i}$ and $u_{i \text { are the }}$ designed parameters that are assigned during the training algorithm of the ANFIS. Output of each node in every layer is denoted by $O_{i}^{1}$ where ${ }^{i}$ specifies the neuron number of the next layer and $l$ is the layer number. The performance of each layer is described in the following:

Layer1(Fuzzification Layer): Each node in this layer is an adaptive node and outputs of these nodes are given by:

$$
\begin{aligned}
& O_{i}^{1}=\mu_{A_{i}}(c v) \\
& O_{i}^{1}=\mu_{B_{i}}(e c) \\
& O_{i}^{1}=\mu_{C_{i}}(e x) \\
& O_{i}^{1}=\mu_{D_{i}}(o t) \\
& i=1,2,3,4
\end{aligned}
$$

Where $\mu_{A_{i}}(c v), \mu_{B_{i}}(e c), \mu_{C_{i}}(e x)$ and $\mu_{D_{i}}(o t)$ are membership functions that determine the degree to which the given $c v, e c, e x_{\text {and }} o t$ satisfy the quantifiers $A_{i}, B_{i}$, $C_{i \text { and }} D_{i}$

Layer2(Product Layer): In this layer, each node is a fixed node and determines the firing strength of the related rule.

$$
O_{i}^{2}=\omega_{i}=\mu_{A_{i}}(c v) \mu_{B_{i}}(e c) \mu_{C_{i}}(e x) \mu_{D_{i}}(o t)
$$


Layer3 (Normalized Layer): In this layer, every node is a circle node and computes the ratio of firing strength of each rule to the total number of rules to obtain the so-called normalized firing strength.

$$
O_{i}^{3}=\bar{\omega}_{i}=\frac{\omega}{\omega_{1}+\omega_{2}+\omega_{3}+\varpi_{4}}
$$

Layer4 (De-Fuzzification Layer): The output of each adaptive node in this layer is:

$$
O_{i}^{4}=\bar{\omega}_{i} f_{i}=\bar{\omega}_{i}\left(p_{i} c v+q_{i} e c+s_{i} e x+t_{i} o t+u\right)
$$

Parameters $p_{i}, q_{i}, s_{i}, t_{i}$ and $u$ are called as consequence parameters.

Layer5 (Output Layer): Final layer, presented with a circle node, calculates the summation of all incoming signals.

$$
O_{i}^{5}=\frac{\sum_{i=1}^{4} \omega_{i} f_{i}}{\sum_{i=1}^{4} \omega_{i i}}
$$

The training efficiency is improved by employing a hybrid learning algorithm to justify the parameters of input and output membership functions. The two parameter sets in the ANFIS architecture that require tuning are the antecedent parameters (the parameters associated with the input membership functions) and the consequent parameters (the parameters associated with the output membership functions). The output of ANFIS will be a linear combination of the consequent parameters which is the premise parameters that can be assumed as fixed. So, the output can be written as:

$$
f=\bar{\omega}_{1} f_{1}+\bar{\omega}_{2} f_{2}+\bar{\omega}_{3} f_{3}+\bar{\omega}_{4} f_{4}
$$

By substituting (8) into (10), the output can be rearranged as follows,

$$
\begin{gathered}
f=\left(\bar{\omega}_{1} c v\right) p_{1}+\left(\bar{\omega}_{1} e c\right) q_{1}+\left(\bar{\omega}_{1} e x\right) s_{1}+\left(\bar{\omega}_{1} o t\right) t_{1}+\left(\bar{\omega}_{1}\right) u_{+} \\
\left(\bar{\omega}_{2} c v\right) p_{2}+\left(\bar{\omega}_{2} e c\right) q_{2}+\left(\bar{\omega}_{2} e x\right) s_{2}+\left(\bar{\omega}_{2} o t\right) t_{2}+\left(\bar{\omega}_{2}\right) u_{+} \\
\left(\bar{\omega}_{3} c v\right) p_{3}+\left(\bar{\omega}_{3} e c\right) q_{3}+\left(\bar{\omega}_{3} e x\right) s_{3}+\left(\bar{\omega}_{3} o t\right) t_{3}+\left(\bar{\omega}_{3}\right) u_{+}
\end{gathered}
$$

$$
\left(\bar{\omega}_{4} c v\right) p_{4}+\left(\bar{\omega}_{4} e c\right) q_{4}+\left(\bar{\omega}_{4} e x\right) s_{4}+\left(\bar{\omega}_{4} o t\right) t_{4}+\left(\bar{\omega}_{4}\right) u
$$

So, the consequent parameters can be adjusted by the least square method. On the other hand, if consequent parameters are fixed, the premise parameters can be adjusted by the gradient descent method. ANFIS utilizes hybrid learning algorithm in which the least square method is used to identify the consequent parameters in the forward pass and the gradient descent method is applied to determine the premise parameters in the backward pass. Hence the ANFIS system is generated for the regions in order to identify the updated building

\subsection{Detection of New Building}

In this phase, the identified uncommon spots $B$ is utilized to detect whether they are building or not. In this case, the uncommon spots $B$ are extracted with the region properties convex area, eccentricity, extent and the orientation. Once the region properties are identified then they are given as input to the ANFIS for identifying whether the identified spot is new building or not. The value of the ANFIS is checked whether they are greater than the threshold 0.5 then the identified uncommon spot is the building which is updated newly rather than the current version. The identified new building is marked in the original current version image which indicates that, that is the building newly updated.

\section{RESULTS AND DISCUSSION}

Updating building database is the main process in order to identify the newly built buildings. Here we utilize 3 ANN in order to identify the traits here the road, vegetation and the building and the identified traits are trained the ANFIS for the further process. In the identification of building database which can be utilized in order to spot the newly updated buildings and the work has been implemented in the working platform (MATLAB 7.11). The obtained results are discussed in the upcoming sessions of this section.

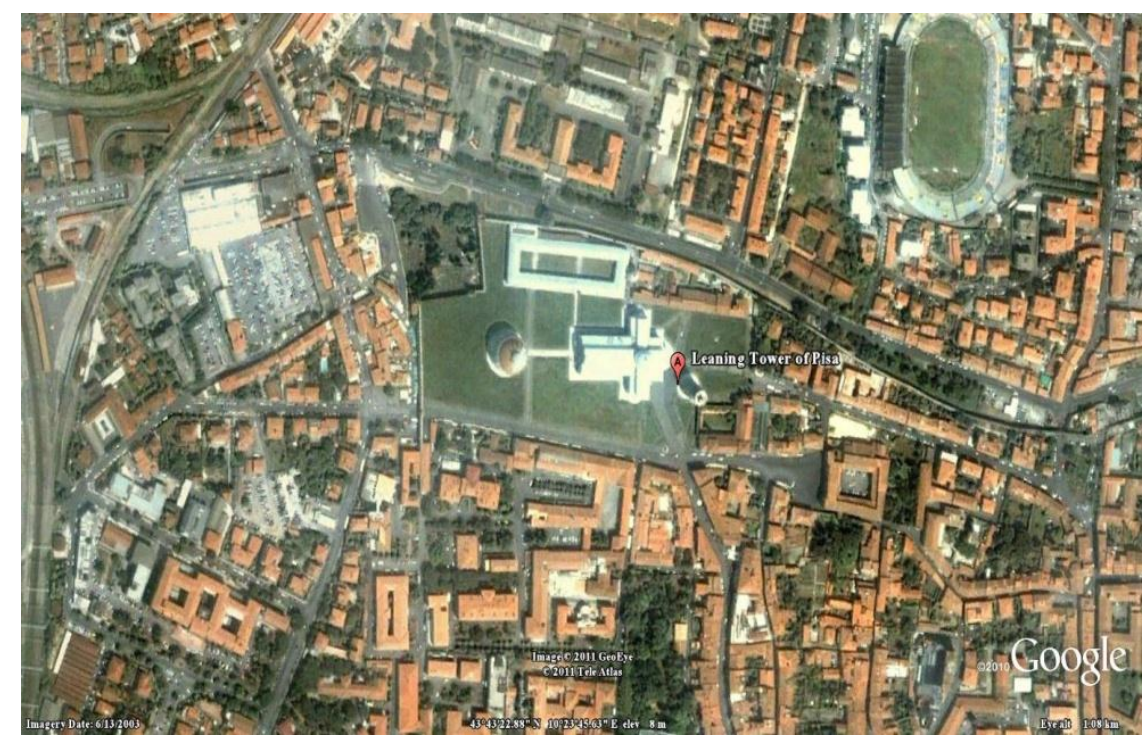

Fig 2: Original Image of the leaning tower of Pisa in the year 2003 


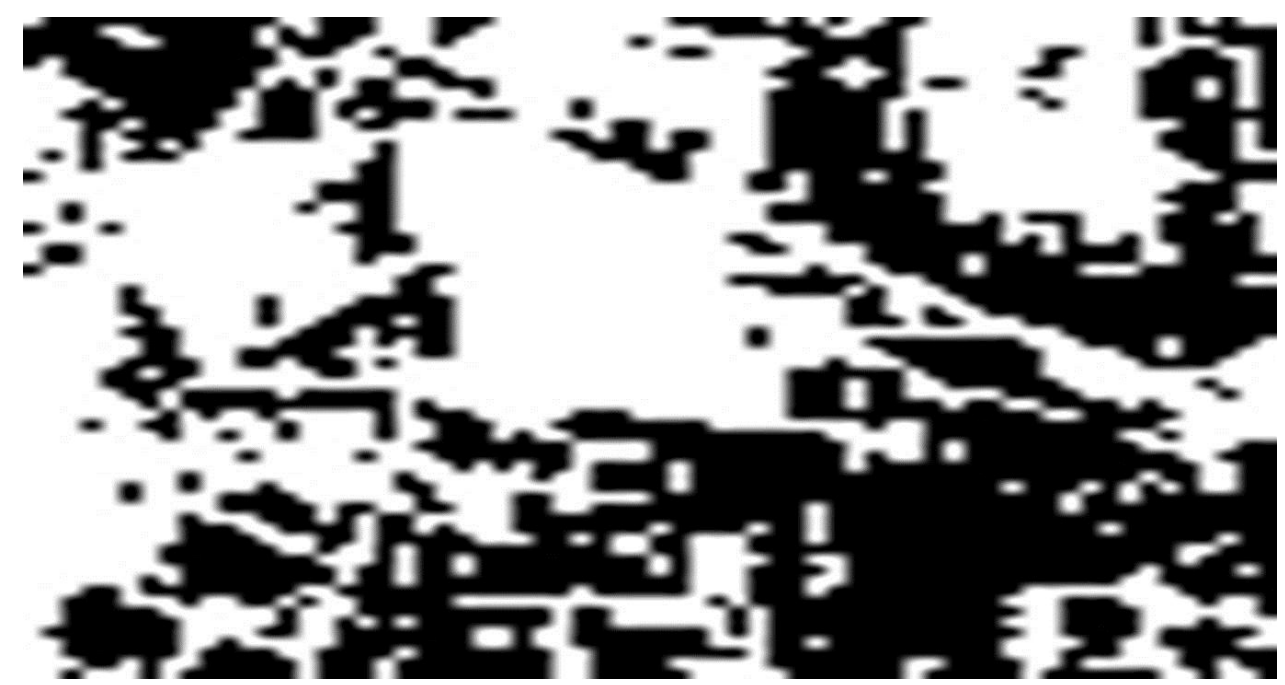

Fig 3: Buildings in the 2003 image

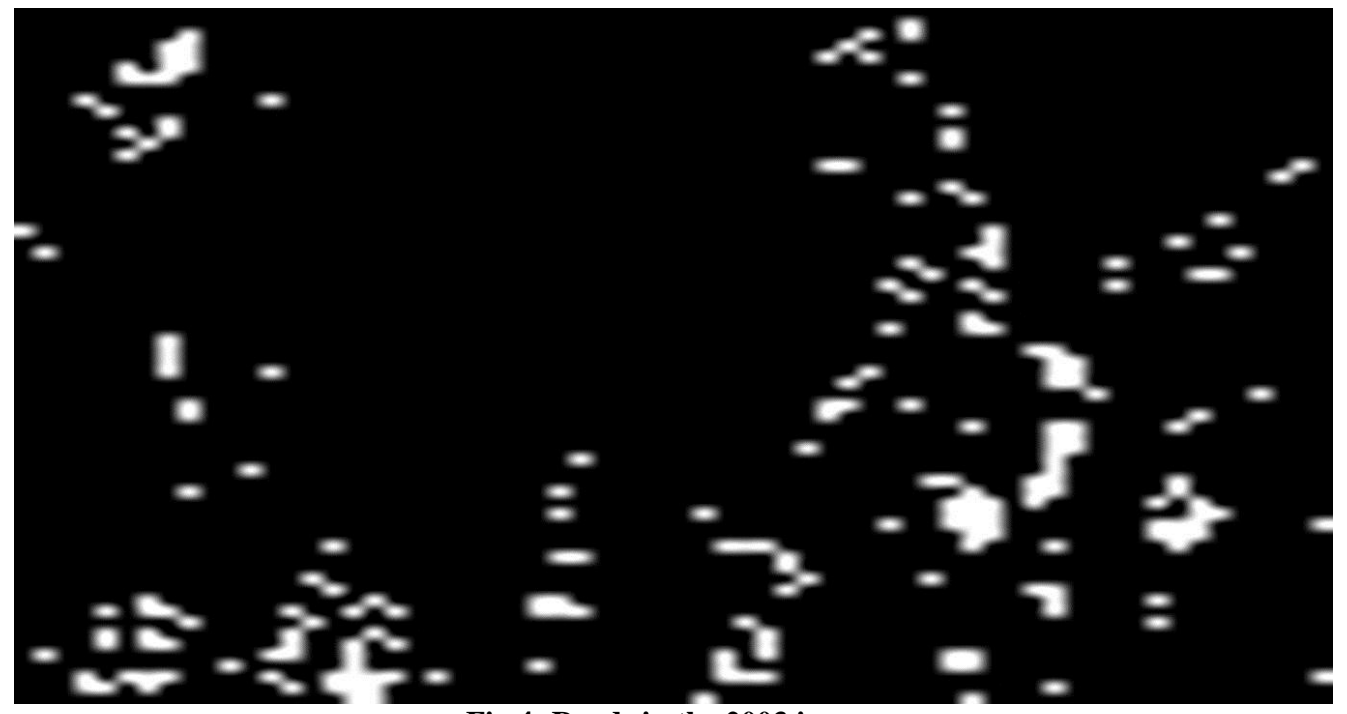

Fig 4: Roads in the 2003 image

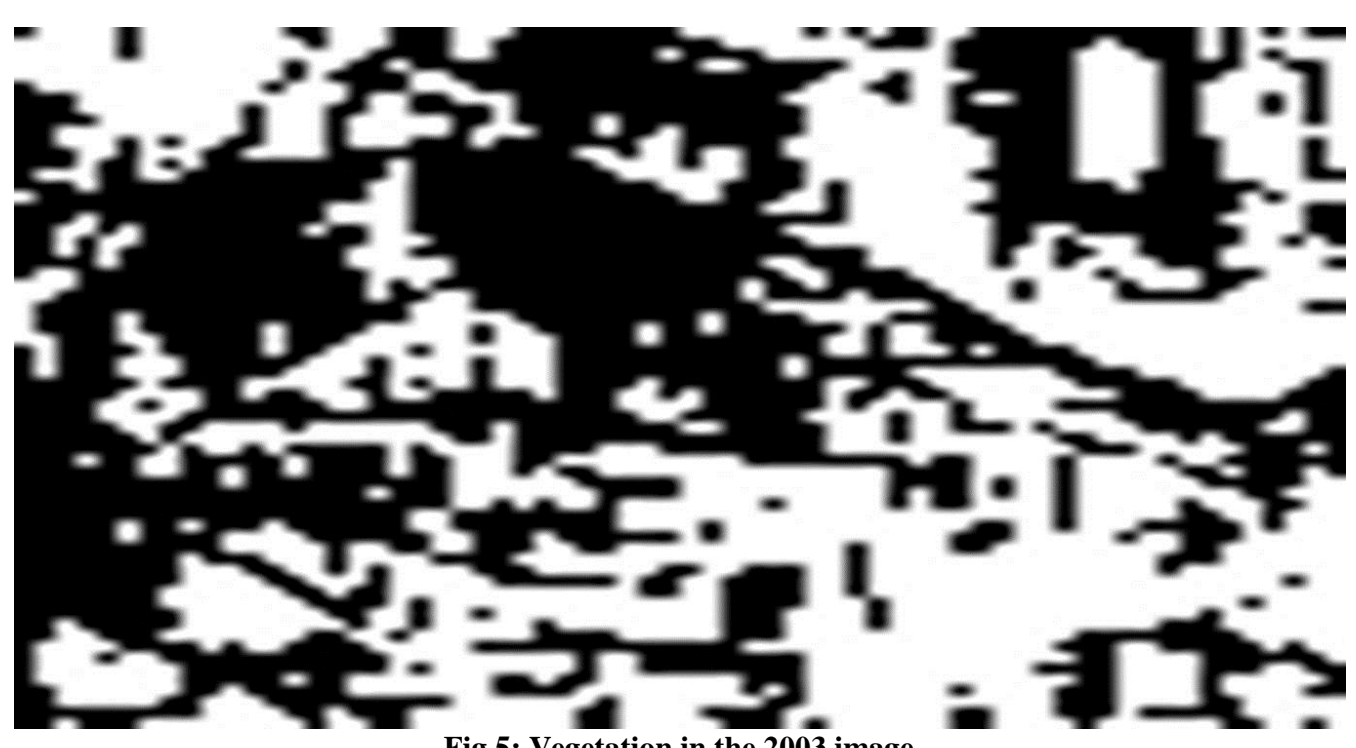

Fig 5: Vegetation in the 2003 image 


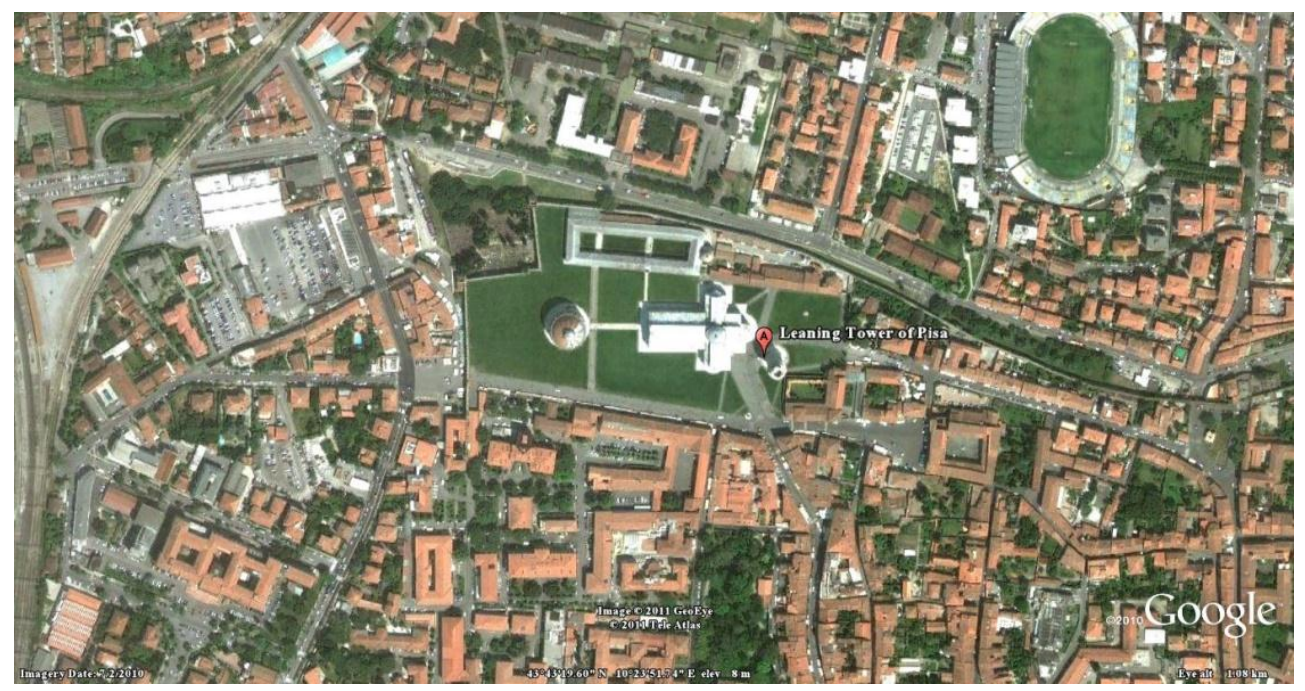

Fig 6: Original Image of the leaning tower of Pisa in the year 2010

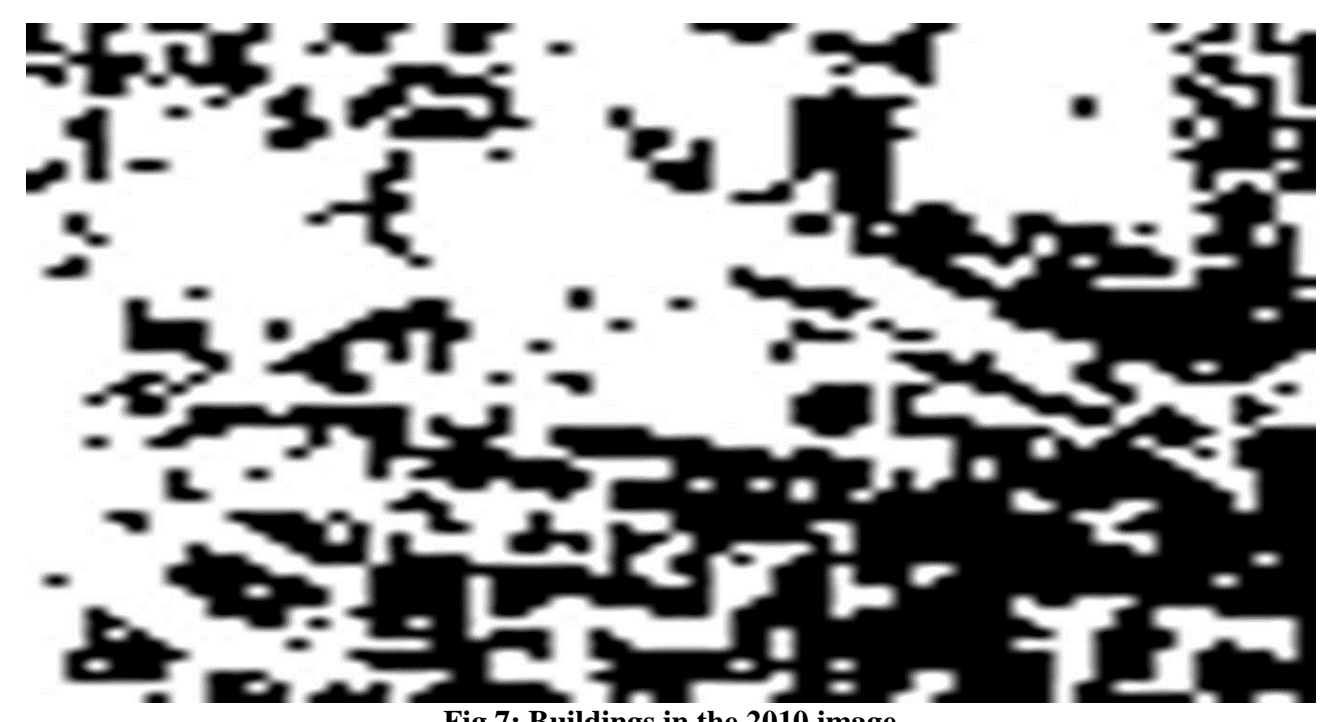

Fig 7: Buildings in the 2010 image

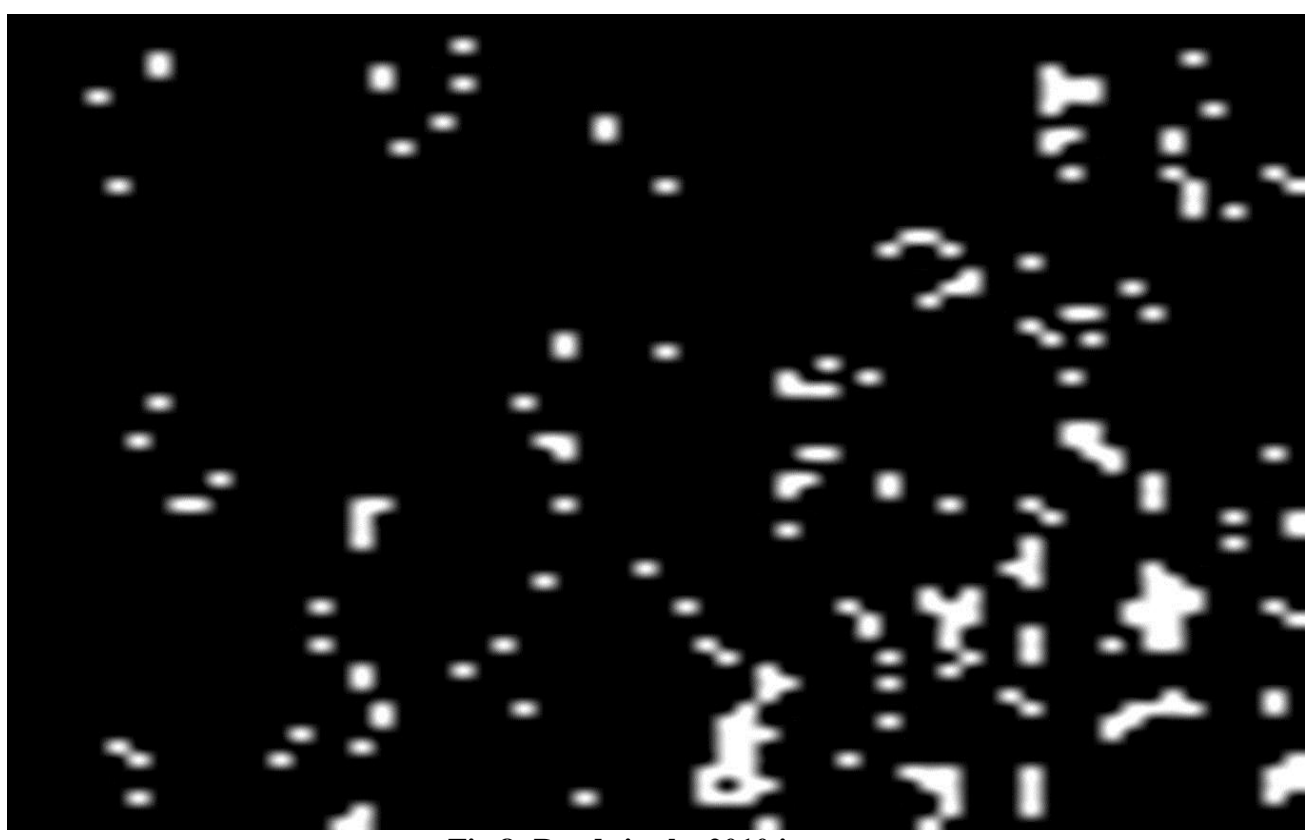

Fig 8: Roads in the 2010 image 

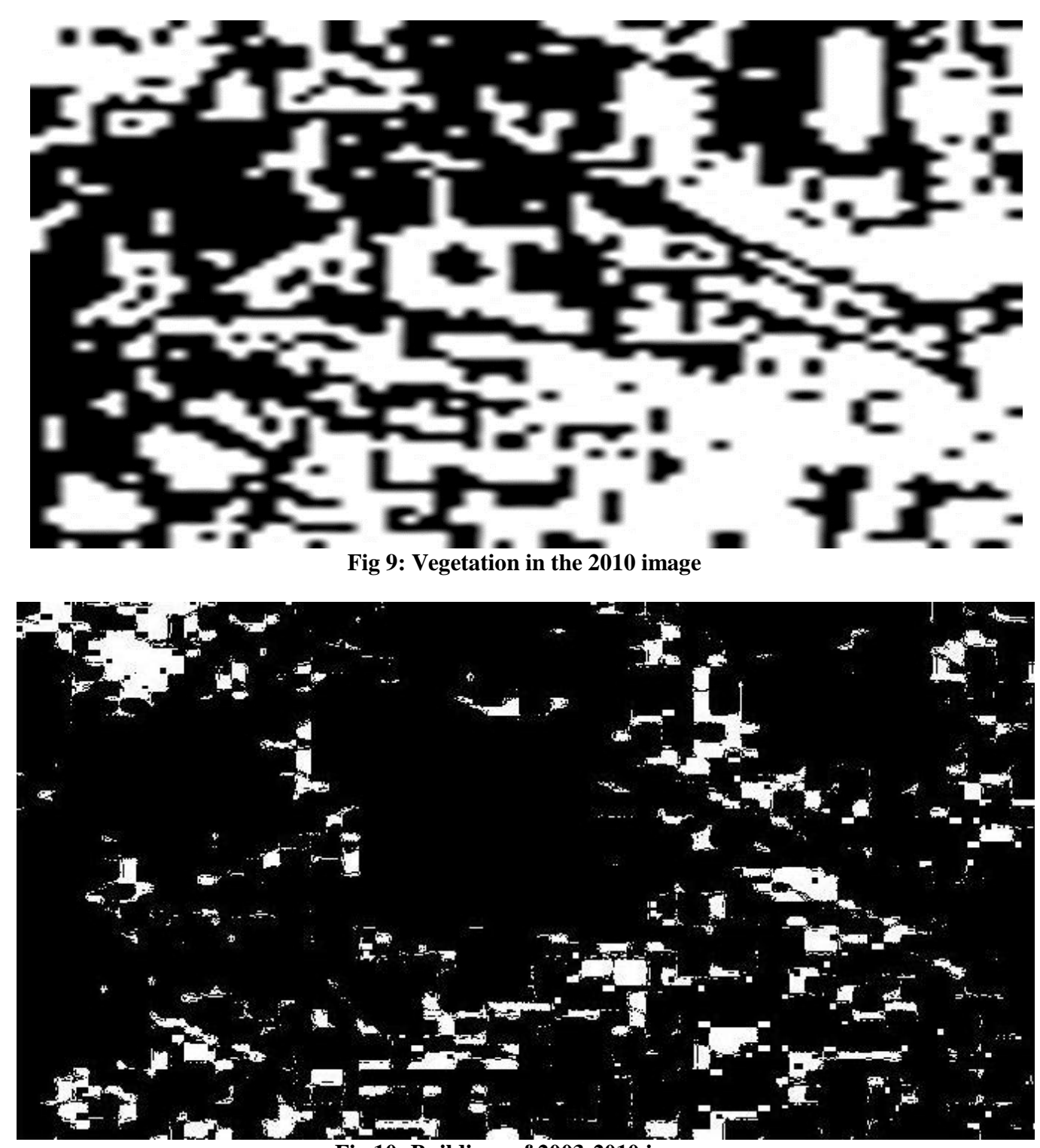

Fig 10: Buildings of 2003-2010 images

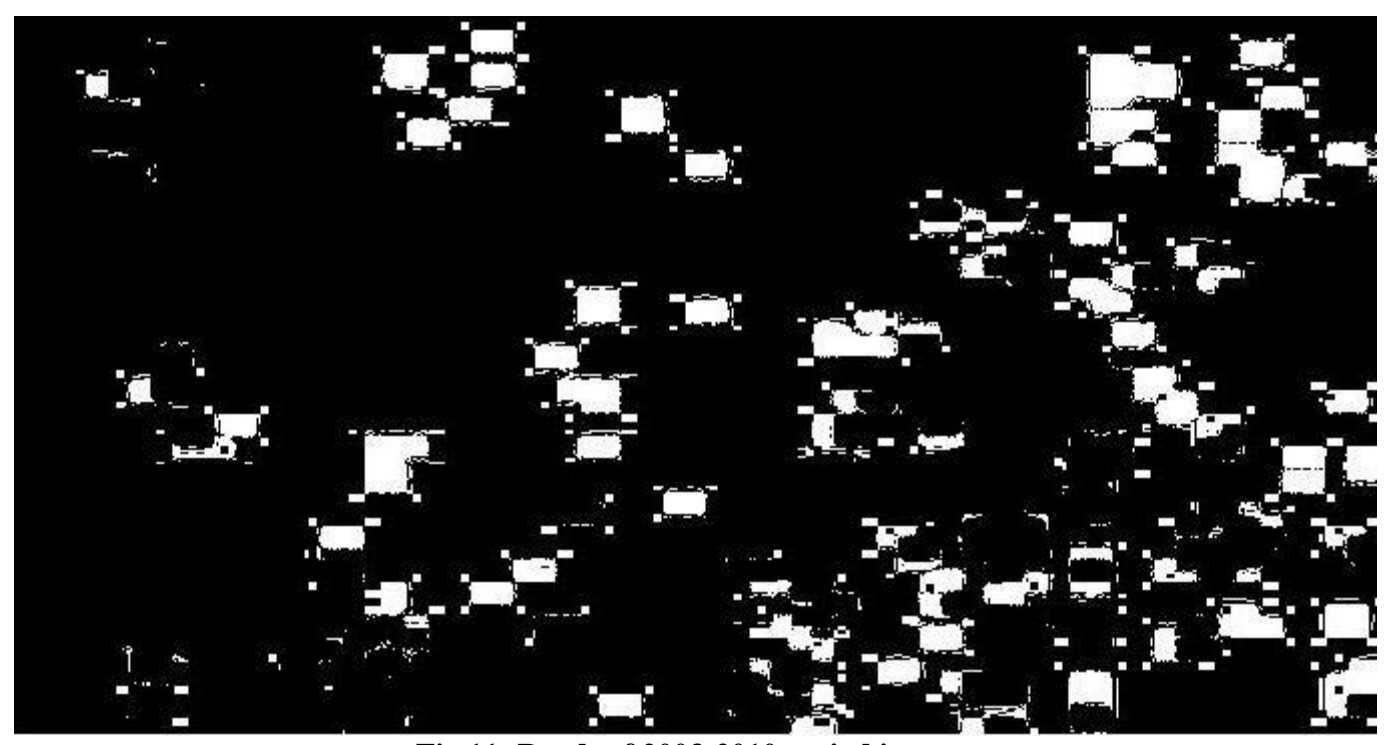

Fig 11: Roads of 2003-2010 period images 


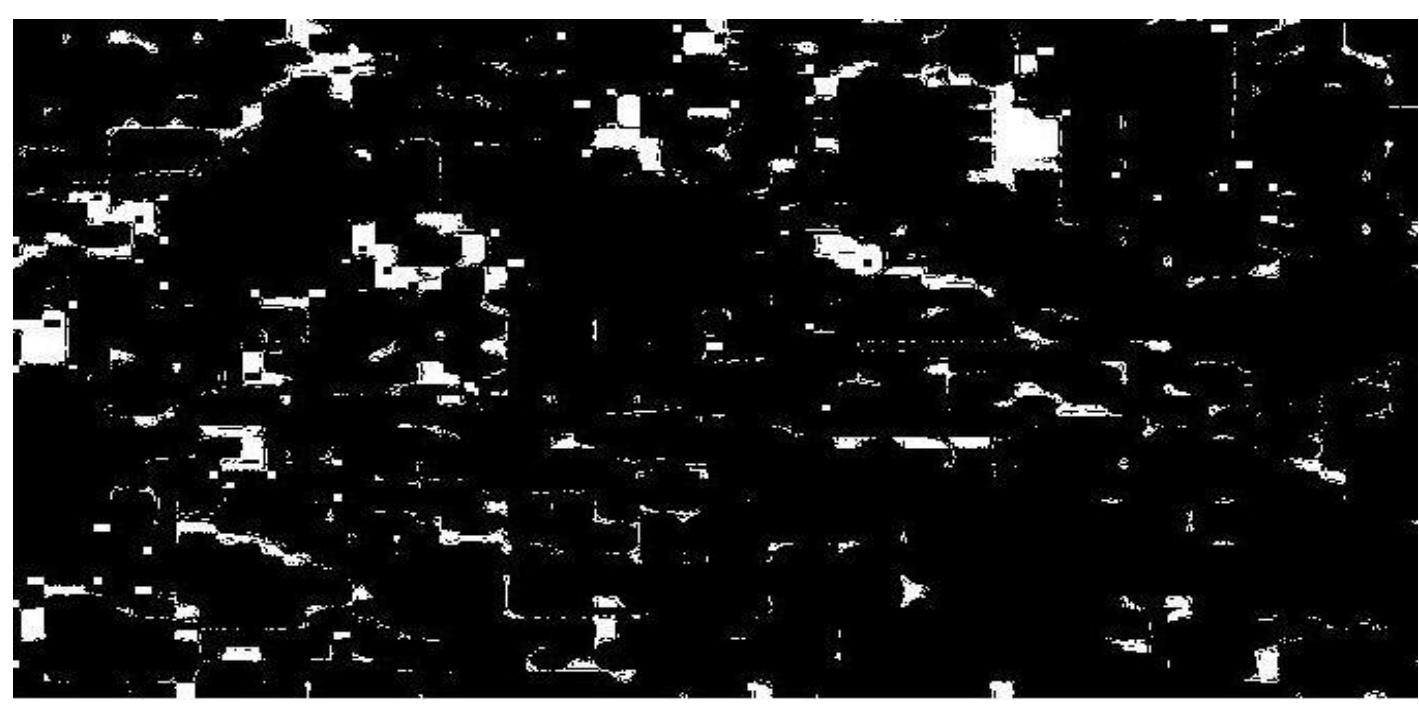

Fig 12: Vegetation in the 2003-2010 period images

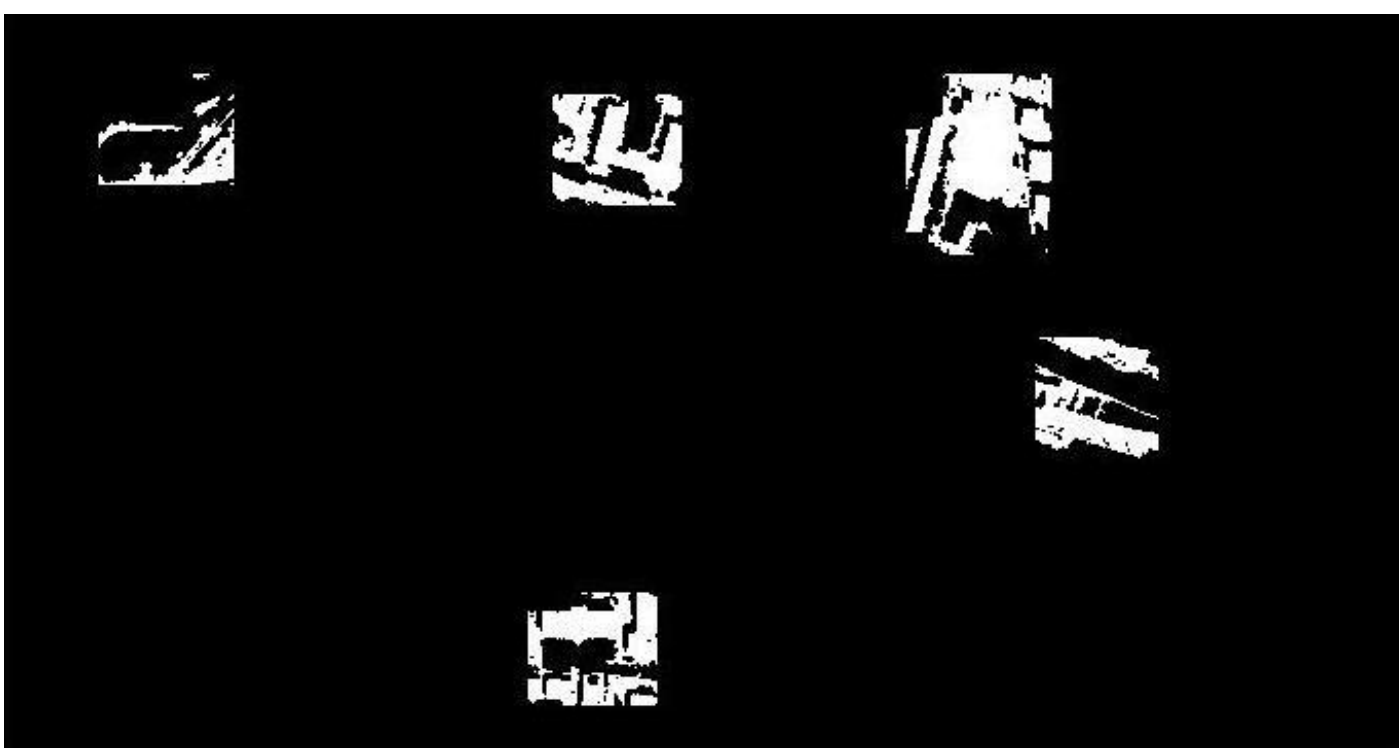

Fig 13: Updated buildings than 2003 spotted in a mask

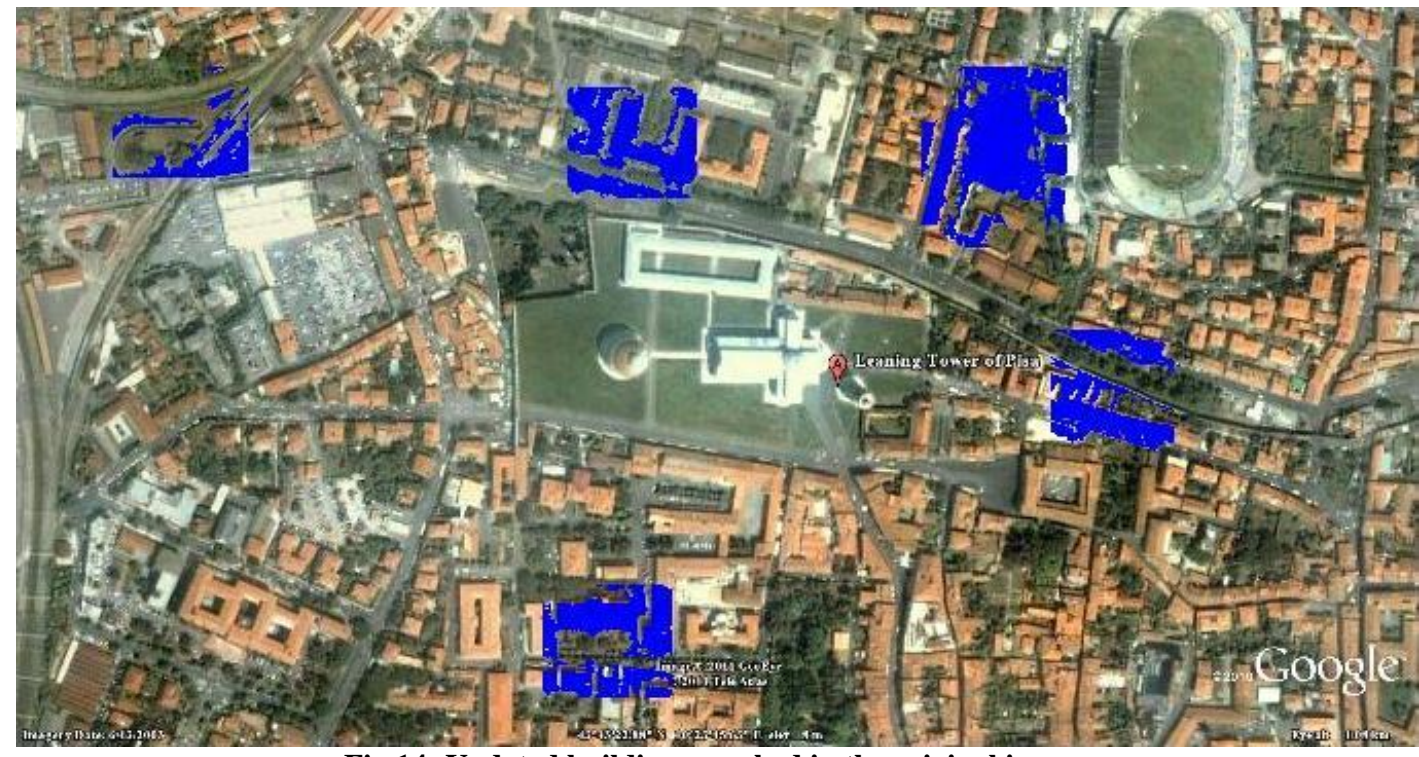

Fig 14: Updated buildings marked in the original image 


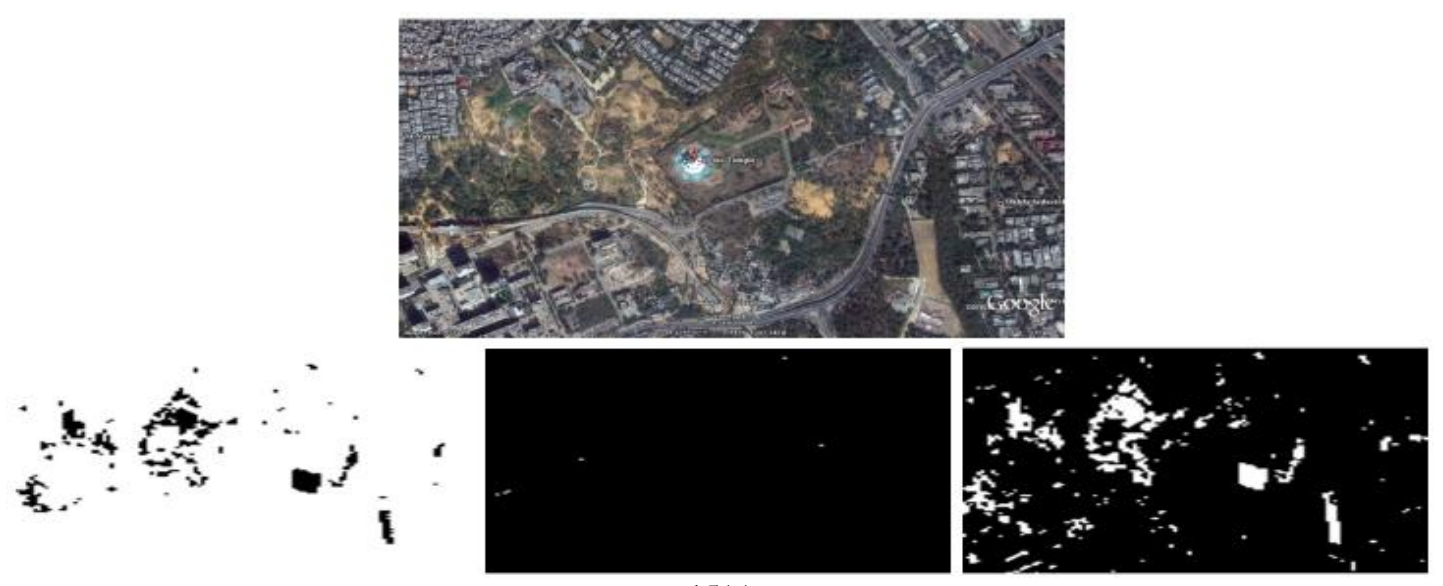

15(a)

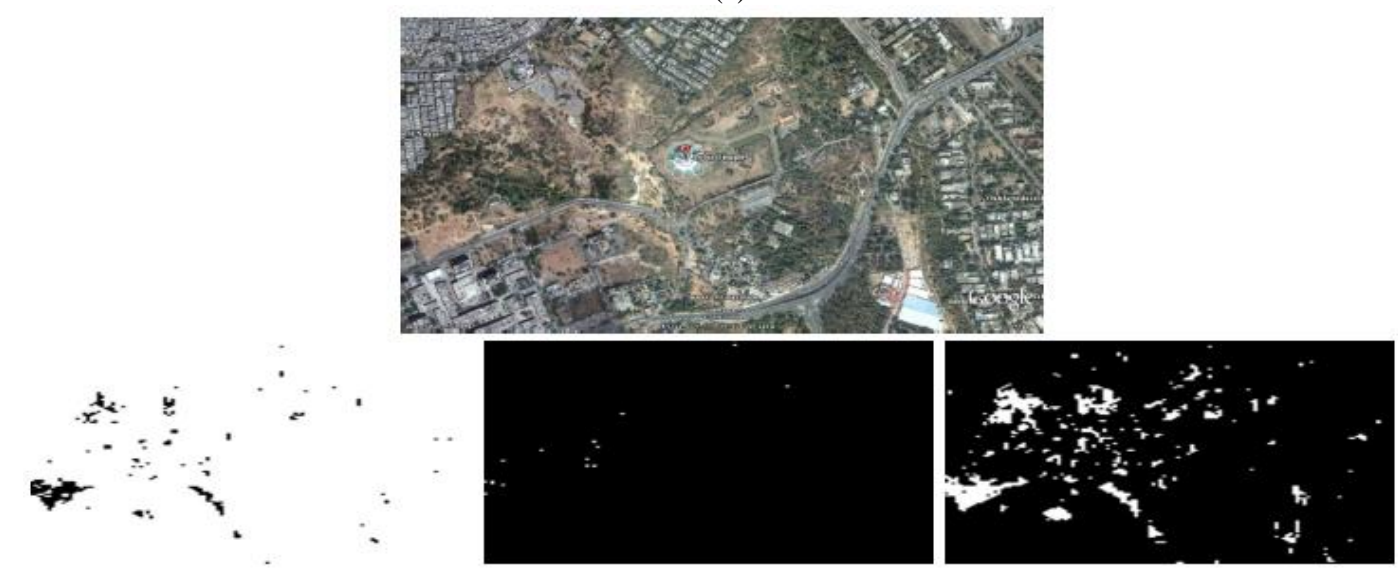

$15(b)$
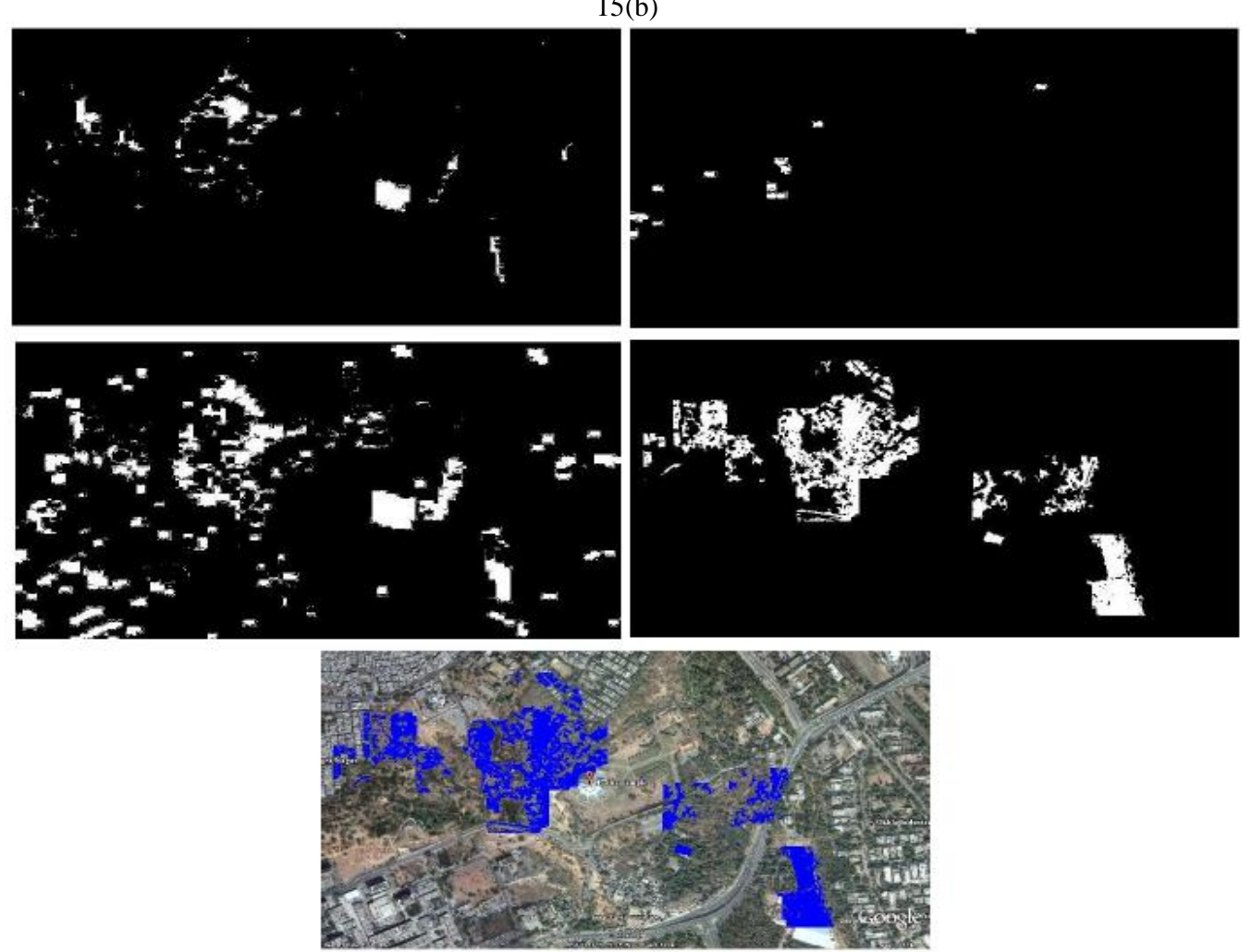

(c)

Fig 15: Images of 2008-2010 (a) 2008 image's traits building, road and vegetation, (b) 2010 image's traits building, road and vegetation 
Table 1. Performance comparison of proposed with the existing work [28]

\begin{tabular}{|l|c|c|c|c|}
\hline \multirow{2}{*}{ Measure } & \multicolumn{3}{|c|}{ Proposed Method } & Exisisting \\
& & I28] \\
\cline { 2 - 4 } & Image 1 & Image 2 & Image 3 & \\
\hline Correctness & $46.66 \%$ & $51.35 \%$ & $71.428 \%$ & $41.6 \%$ \\
\hline Mistreated & $53.33 \%$ & $48.648 \%$ & $28.571 \%$ & $58.4 \%$ \\
\hline
\end{tabular}

The above images are the results of the proposed work with different images of different period. Fig 2 depicts the original image of the year 2003 which is leaning tower of Pisa. Fig 3, 4 and 5 depicts the traits building, roads and vegetation respectively. Fig 6 depicts the original image of the same spot of the period 2010. Figs 7, 8 and 9 depicts the traits building, roads and the vegetation marked images respectively. Figs 10, 11 and 12 depict the combined traits of the different periods 2003-2010 which are the building, road and the vegetation. Fig 13 is the updated buildings analyzed with the aid of our proposed work. Fig 14 showed the updated buildings marked in the original image of the period 2010. Fig 15 depicts another image evaluated with our proposed work. Table 1 details the performance of our work compared with the existing work [28].

\section{CONCLUSION}

An effective identification for the updated building has been done with the aid of the ANN and ANFIS. Initially, the two different period building images have been taken which are belongs to same place. Both images are extracted with the traits road, building and the vegetation with the aid of 3 different trained ANNs. Once the traits are identified, then they have been verified in the region wise feature detector. Then the traits of the different periods are analyzed in order to identify the unique spot in the recent image. Once these processes have been completed, then the identified unique spot is analyzed whether it is building or not once identified then they have been marked in the original image. Hence the performance measure has been analyzed and compared with the existing work. The significance of the method can be seen from the results that the correctness of the image from proposed method is relatively higher than the existing method, whereas the mistreated results are relatively lesser than the existing method.

\section{REFERENCES}

[1] Xuelian Meng, Nate Currit,Le Wang," MorphologyBased Building Detection From Airborne Lidar Data", proceeding of the Conference on ASPRS Annual Portland, Oregon, April 28 - May 2, 2008

[2] Mohammad Awrangjeb, Chunsun Zhang and Clive S. Fraser," Improved Building Detection Using Texture Information", International Archives of Photogrammetry, Remote Sensing and Spatial Information Sciences, Vol.38, 2011

[3] Dong-Min Wooa, Quoc-Dat Nguyena, Quang-Dung Nguyen Trana, Dong-Chul Parka, Young-Kee Jungb,"Building Detection And Reconstruction From Aerial Images", The International Archives of the Photogrammetry, Remote Sensing and Spatial Information Sciences. Vol. XXXVII, Beijing 2008

[4] Cheng-Kai Wang, Pai-Hui Hsu,"Building Detection and Structure Line Extraction from Airborne LiDAR Data",
Journal of Photogrammetry and Remote Sensing,Vol. 12, No.4, pp. 365-379, December 2007

[5] Xuelian Meng, Le Wang, and Nate Currit,"Morphologybased Building Detection from Airborne Lidar Data", Photogram metric Engineering \& Remote Sensing, Vol. 75, No. 4,pp. 437-442, April 2009

[6] Konstantinos Karantzalos and Nikos Paragios, "Recognition-Driven 2D Competing Priors towards Automatic and Accurate Building Detection", Journal of Tgars, pp.1-11, 2008

[7] D. Koc San and M. Turker ,"Building Extraction From High Resolution Satellite Images Using Hough Transform", International Archives of the Photogrammetry, Remote Sensing and Spatial Information Science, Volume XXXVIII, No. 8, Kyoto Japan 2010

[8] Xiaoying Jin, "Automated Building Extraction from High-Resolution Satellite Imagery in Urban Areas Using Structural, Contextual, and Spectral Information, "EURASIP Journal on Applied Signal Processing, Vol.14, pp.2196-2206,2005

[9] Masoud S. Nosrati and Parvaneh Saeedi, "A Novel Approach For Polygonal Rooftop Detection In Satellite/Aerial Imageries, "proceeding of the IEEE International Conference on Image Processing,pp.17091712,2009

[10] Mostafa Kabolizade, Hamid Ebadi, Salman Ahmadi, "An Improved Snake Model for Automatic Extraction of Buildings from Urban Aerial Images and LiDAR Data Using Genetic Algorithm",IAPRS, Vol. XXXVIII, No. 3B - Saint-Mandé, France, September 1-3, 2010

[11] J. D. Wegner, A. O. Ok, A. Thiele, F. Rottensteiner a, U. Soergel, "Urban Building Detection From Optical And Insar Features Exploiting Context", APRS, Vol. XXXVIII, No. 3A, Saint-Mandé, France, September 1-3, 2010

[12] Sherif Elghazali, "Performance of Quickbird Image and Lidar Data Fusion for 2d/3d City Mapping, "Australian Journal of Basic and Applied S ciences,Vol.5,No.11, pp.1588-1600, 2011

[13] Konstantinos Karantzalos and Demertre Argialas, "A Region-based Level Set Segmentation for Automatic Detection of Man-made Objects from Aerial and Satellite Images", Photogrammetric Engineering \& Remote Sensing Vol. 75, No. 6, pp. 667-677, June 2009

[14] H. Sportouche, F. Tupin and L. Denise, "Building Detection by Fusion of Optical and SAR Features in Metric Resolution Data", proceeding of the IEEE International Geoscience \& Remote Sensing Symposium, pp.769-772, 2009 
[15] H. Sportoucheand L. Denise, "Building Detection And Height Retrieval In Urban Areas In The Framework Of High Resolution Optical And Sar Data Fusion", proceeding of the IEEE International Geoscience \& Remote Sensing Symposium,pp.3660-3663, 2010

[16] Mohammad Awrangjeb, Mehdi Ravanbakhsh and Clive S. Fraser, "Building Detection from Multispectral Imagery And Lidar Data Employing A Threshold-Free Evaluation System ", IAPRS, Vol. XXXVIII, No. 3A Saint-Mandé, France, September 1-3, 2010

[17] Beril Sırmaçek and Cem Ünsalan, "A Probabilistic Framework to Detect Buildings in Aerial and Satellite Images Beril", IEEE Transactions On Geoscience And Remote Sensing, Vol. 49, No. 1, January 2011

[18] H. G"okhan Akc,ay, Selim Aksoy, "Building Detection Using Directional Spatial Constraints, "proceeding of the IEEE International conferences on Geoscience and Remote Sensing Symposium (IGARSS), 2010

[19] Liang-Chien Chen and Li-Jer Lin, "Detection of building changes from aerial images and light detection and ranging (LIDAR) data, "Journal of Applied Remote Sensing, Vol. 4 , November 2010

[20] Andreas Jochema, Bernhard ofleb, Markus Hollausb, Martin Rutzingerc, "Object Detection In Airborne Lidar Data For Improved Solar Radiation Modeling In Urban Areas", IAPRS , Vol. XXXVIII, Paris, France, September 1-2, 2009

[21] Beril Sirmacek, Cem Unsalan, "Urban Area Detection Using Local Feature Points and Spatial Voting, " IEEE geoscience and remote sensing letters, VOL. 7, NO. 1, pp. no.146-150, January 2010.

[22] Beril Sirmacek, Cem Unsalan, " A Probabilistic Framework to Detect Buildings in Aerial and Satellite
Images , " IEEE geoscience and remote sensing letters, VOL. 49, NO. 1, pp. no.211-221, January 2011.

[23] Lau Bee Theng, " Automatic Building Extraction from Satellite Imagery, Vol.5, " November 2006.

[24] Beril Sirmacek and Cem Unsalan "A Probabilistic Framework to Detect Buildings in Aerial and Satellite Images", IEEE transactions on geoscience and remote sensing, Vol. 49, No. 1, pp.211-221, 2011.

[25] Beril Sirmacek and Cem Unsalan "Urban-Area and Building Detection Using SIFT Keypoints and Graph Theory", IEEE transactions on geoscience and remote sensing, Vol. 47, No. 4, pp.1156-1167, 2009.

[26] Xian-Zhang Pan, Qi-Guo Zhao, Jie Chen, Yin Liang and Bo Sun "Analyzing the Variation of Building Density Using High Spatial Resolution Satellite Images, , SensorsVol.8, No.4, pp. no. 2541-2550, 2008.

[27] Mohammad Awrangjeb, Mehdi Ravanbakhsh and Clive S. Fraser, "Building detection from multispectral imagery and lidar data employing a threshold-free evaluation system," International Archives of Photogrammetry, Remote Sens. \& Spatial Information Sciences, Vol.38, pp.no.49-54, Sep.2010

[28] Champion1, Stamon and Pierrot-Deseilligny, "Automatic GIS Updating from High Resolution Satellite Images", In proceedings of IAPR Conference on Machine Vision Applications, pp.374-377,2009

[29] Ayyoub Rezaeeian, Aghil Yousefi-Koma, Behrouz Shast and Alireza Doosthoseini, "ANFIS Modeling and Feed forward Control of Shape Memory Alloy Actuators", International Journal Of Mathematical Models And Methods In Applied Sciences,Vol.2, No.2, pp.228-235, 2008 